\title{
结合影子障碍物和 ORCA 模型的人群仿真方法
}

何高奇 ${ }^{1,2}$ ，江东旭 ${ }^{1}$ ，金祎 1 ，陈琪 ${ }^{1}$ ，卢兴见 $1,3^{*}$ ，徐明亮 4

1. 华东理工大学计算机科学与工程系, 上海 200237

2. 华东师范大学地理信息科学教育部重点实验室, 上海 200241

3. 上海交通大学智慧城市协同创新中心, 上海 200240

4. 郑州大学信息工程学院, 郑州 450000

* 通信作者. E-mail: luxj@ecust.edu.cn

收稿日期: 2017-11-24; 接受日期: 2017-12-12；网络出版日期: 2018-03-16

国家自然科学基金 (批准号: 61602175, 61472370, 61672469)、地理信息科学教育部重点实验室基金 (批准号: KLGIS2015A05)、 上海市软件和集成电路产业发展专项资金 (批准号: 150809)、国家重点研究计划 (批准号: 2017YFC0804401) 和北京航空航天大学 虚拟现实技术与系统国家重点实验室开放课题 (批准号: BUAA-VR-16KF-07) 资助项目

摘要 人群仿真已经成为疏散演练、安全监控等重要应用领域的关键支撑技术, 但是复杂的个体心 理、个性特征, 以及个体间的相互影响对人群仿真的真实感提出了挑战. 本文从用户的安全心理特 征和运动速度特征出发, 研究个体在拐角处的运动轨迹和避碰行为的真实感. 首先, 将影子障碍物 模型分别转化为 ORCA (optimal reciprocal collision avoidance) 半平面和期望速度, 分别提出了两种 影子障碍物和 ORCA 模型的结合方法. 然后, 通过提出影子障碍物的手动生成方法, 处理任意角度 的拐角以及一些非封闭墙面的情况. 基于上述方法, 融合行为模拟和物理模拟技术, 提出基于速度 的人群仿真框架, 并对复杂场景以及大规模人群进行实验分析. 最后, 将上述方法应用在地铁站环境 的复杂场景, 并采用 Unity3D 引擎进行真实感渲染, 在仿真效果和计算效率两方面与现有的社会力 模型进行比较. 实验结果表明, 本文提出的结合影子障碍物和 ORCA 模型的人群仿真方法计算效率 高、真实感强, 具有较好的应用前景.

关键词人群仿真, 影子障碍物模型, ORCA 模型, Unity3D, 真实感渲染

\section{1 引言}

人群仿真是通过研究人群在各种环境下的运动特征与规律, 建立人群运动的仿真模型, 并在计算 机虚拟环境中逼真地展示人群的运动过程 ${ }^{[1]}$. 近年来, 人群仿真不再仅仅局限于计算机动画, 而是已 经成为众多应用领域中的研究焦点 ${ }^{[2]}$, 并广泛应用于市政建设中公共场所的布局模拟 ${ }^{[3]}$ 、疏散演练中 的恐怖袭击模拟 ${ }^{[4]}$, 以及军事仿真中的战略战术模拟 [5] 等重要场景. 人群仿真方法大致可分为基于 连续性和基于智能体两个层面 ${ }^{22]}$, 其中基于智能体层面的局部行为真实感模拟已是人群仿真的重要方

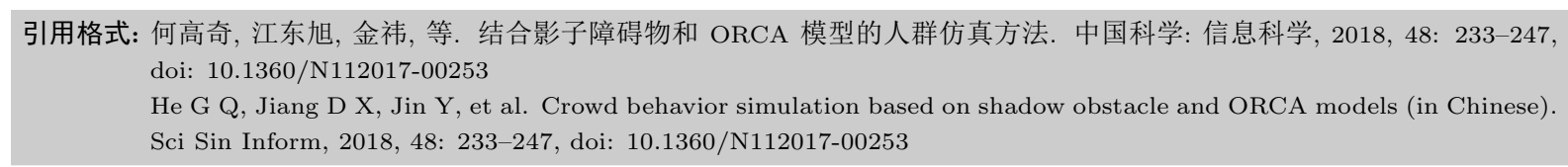


向之一 ${ }^{[6]}$. 智能体行为模拟中比较经典的方法有基于社会力的仿真模型 ${ }^{[7]}$ 和基于速度的仿真模型 ${ }^{[4]}$, 但是这些基于智能体的仿真模型均存在一定的行人抖动现象 ${ }^{[2,7,8]}$, 这成为仿真个体行为控制模型研 究中的一项重要难题.

为了获得较强的行为真实感, 传统的人群仿真算法通常采用基于最短路径原则 [9] 来设定行人的 运动轨迹. 然而该方法生成的个体轨迹不够平滑、拐角处轨迹不够自然, 并且个体在拐角处极易发生 碰撞 ${ }^{[10]}$. 为了构建行人在拐角处逼真的轨迹, 影子障碍物模型 ${ }^{[11]}$ 将行人的自我安全意识因素加入到 行人运动的研究中, 使得生成的轨迹较最短路径而言更具有多样性和真实感, 但是该模型同样在拐角 处存在行人碰撞的问题.

为使人群仿真中的个体在拐角处具有真实的运动轨迹, 并且个体之间具有良好的避碰行为, 本文 将结合安全心理特征和运动速度特征, 提出一种结合影子障碍物和 ORCA (optimal reciprocal collision avoidance) 模型 ${ }^{[12]}$ 的人群仿真方法. 本文贡献点主要有以下 3 个方面:

(1) 将影子障碍物模型转化为 ORCA 半平面和期望速度, 提出了两种影子障碍物和 ORCA 模型 的结合方法, 使得个体运动具有高度真实感;

(2) 提出影子障碍物的手动生成方法, 从而可以处理任意角度的拐角以及非封闭墙面的情况;

(3) 融合行为模拟和物理模拟技术, 提出基于速度的人群仿真框架, 实现了复杂场景中大规模人群 的行为仿真.

\section{2 国内外研究现状}

人群仿真 [13] 最早起源于动物群体仿真, 相比动物群体而言, 人群的复杂性使得人群的模拟更加 困难. 为了模拟复杂动态场景中的群体运动与避碰行为, 人群仿真方法大致可分为基于连续性和基于 智能体两个层面 ${ }^{[2]}$. 从基于智能体层面来说, 行人体型、避碰速度、个体空间需求等是仿真研究中重 要的参数特征 ${ }^{[14]}$; 从基于连续性层面来说, 行人流被看做一个连续流动的介质, 流量分布、时空演化 特性, 以及自组织行为是人群行为研究的重要角度 ${ }^{[15]}$. 由于当前人群行为仿真更加依赖于基于智能 体的研究, 并且近年来对虚拟个体避碰行为的研究具有显著的进展 ${ }^{[16]}$, 因此将人群仿真行为的研究集 中在基于智能体层面上成为主要趋势.

\section{1 基于智能体的行为控制模型研究}

基于智能体的模型最早可追溯到 Reynold 对于类鸟群的开创性研究 ${ }^{[17,18]}$. 根据研究方法的不同, 基于智能体的行为控制模型大致可以分为两类: 基于社会力的仿真模型和基于速度的仿真模型. 基于 社会力的模型最早由 Helbing 等 ${ }^{[7]}$ 提出, 该研究将人群中的个体看作是拥有质量和速度的粒子, 他们 受到心理力和物理力的混合作用, 但是相对于实际的行人运动, 社会力模型仍然不够真实 ${ }^{[19]}$. 为了提 高仿真个体避碰行为的真实感, Pelechano 等 [20] 引入了情绪因子来模拟恐慌情绪感染对于个体运动 的影响. Moussaid 等 ${ }^{21]}$ 在社会力模型中加入行人的视觉因素作为行人的感知范围, 提高了在多个体 场景中的计算效率和仿真的真实性. 为了解决人群被分开后重建其连贯性的问题, Kremyzas 等 ${ }^{[22]}$ 在 Moussaid 等 [21] 研究的基础上提出了一种社会群体导航的方法, 从全局和局部规划的层次对社会群体 行为进行仿真.

基于速度的模型是近几年提出的行为控制模型, 其中最早提出的是 RVO (reciprocal velocity obstacle) 模型 ${ }^{[23]}$, 该模型主要存在的问题是两个相向而行的个体在选择避碰方向时可能会选择同一侧 
进行避碰, 从而导致碰撞. 为了解决 RVO 中存在的行人抖动问题, Berg 等 ${ }^{[12]}$ 提出了相关的改进模型 ORCA, 并且在 ORCA 模型的基础上引入了视觉因素 ${ }^{[24]}$, 通过考虑周围个体的远近距离来减少碰撞 发生的概率. 基于速度的模型也能够构建行人之间的群体性模型, 其中 Karamouzas 等 ${ }^{[16]}$ 提出了一种 基于速度的成本优化算法来保证避碰的行为仿真, 接着 $\mathrm{Wu}$ 等 ${ }^{[25]}$ 将 Karamouzas 的研究与 $\mathrm{Jan}{ }^{[26]}$ 基 于视觉导航的研究成果结合在一起, 应用在了真实的人群场景中.

\section{2 基于行为真实感的影子仿真研究}

在人群仿真的诸多应用中, 为了使仿真个体在虚拟世界中的行为更加趋近于人类, 仿真个体的行 走路径规划和避碰行为成为提高真实感的重要工作 ${ }^{[16]}$, 其中 Suzuki 等 ${ }^{[27]}$ 研究了仿真个体在条形走 廊的避碰行为, 而 Hashimoto 等 ${ }^{[10]}$ 认为在服务型的社会中, 拐角处的行为真实感研究更为必要. 为了 解决仿真个体在拐角处的避碰行为, Hashimoto 等根据行人的安全心理, 通过调查研究得出了人在拐 角处的一些转弯特性. 在此基础上, He 等 ${ }^{[11]}$ 进一步提出了影子障碍物模型, 即在拐角处产生没有实 体的障碍物, 对转弯时行人的运动轨迹进行修正, 从而提高行人在拐角处运动轨迹的真实感. “影子” 的概念主要是受到 Yeh 等 ${ }^{[28]}$ 的启发, 他们提出了 “复合个体” 的概念, 采用基础个体与复合个体两类 行人来对人群仿真中的多个体行为进行模拟, 实现行人避碰的目的. 基于这一研究, $\mathrm{Xu}$ 等 ${ }^{[29]}$ 提出了 影子交通模型, 在交通异常的点上生成并不存在的车辆, 用该假设的车辆行为来模拟异常的交通行为.

在人群行为仿真的研究中, 还存在许多用来提高人群行为真实感的模型 ${ }^{[30,31]}$. 这些模型相对粗 糙, 仅采用人为的方法生成了转弯曲线, 但是影子障碍物则考虑到了人群心理对行为特性的影响, 生成 的运动轨迹更符合行人的一般决策. 除此之外, 基于速度的 ORCA 模型能够有效地处理仿真个体间 的避碰问题, 并且该模型具有较强的理论基础 ${ }^{[32]}$. 因此将这两种模型进行结合, 能够在保持拐角处行 人运动真实感的同时有效地避免行人发生碰撞, 从而提升了人群仿真中个体轨迹的真实感.

因此, 本文将在现有基于智能体的行为仿真研究基础上, 针对人群仿真研究中存在的拐角处个体 轨迹真实感较差的问题, 提出结合影子障碍物和 ORCA 模型的人群仿真方法, 实现复杂场景中人群运 动行为的高度模拟; 并构建地铁站环境的复杂场景, 在该场景中实现人群仿真模型的应用, 进一步验 证算法的有效性.

\section{3 基于影子障碍物及 ORCA 模型的个体运动仿真方法}

现有的影子障碍物模型可以提高仿真个体在拐角处的行为真实感, ORCA 模型能够模拟人群运动 的避碰行为. 本文以这两个模型为基础, 通过利用影子障碍物模型得出转弯行为的可行速度集合和期 望速度, 以实现对个体运动的高度仿真模拟. 本文的主要工作如图 1 所示.

- 将影子障碍物与 ORCA 模型进行结合, 实现具有更强真实感的速度选择;

- 提出影子障碍物模型的手动生成方法, 根据自定义需求手动创建多边形, 实现非直角场景中的影 子障碍物生成;

- 提出基于速度的人群仿真框架, 实现两套人群仿真算法;

- 对复杂场景及大规模人群的转弯行为进行实验分析, 与基于社会力的模型的仿真效果进行对比.

\section{1 影子障碍物模型}

影子障碍物 ${ }^{[11]}$ 是模拟行人转弯行为的一种方法. 在模拟仿真开始之前, 该方法将选择各向同性 


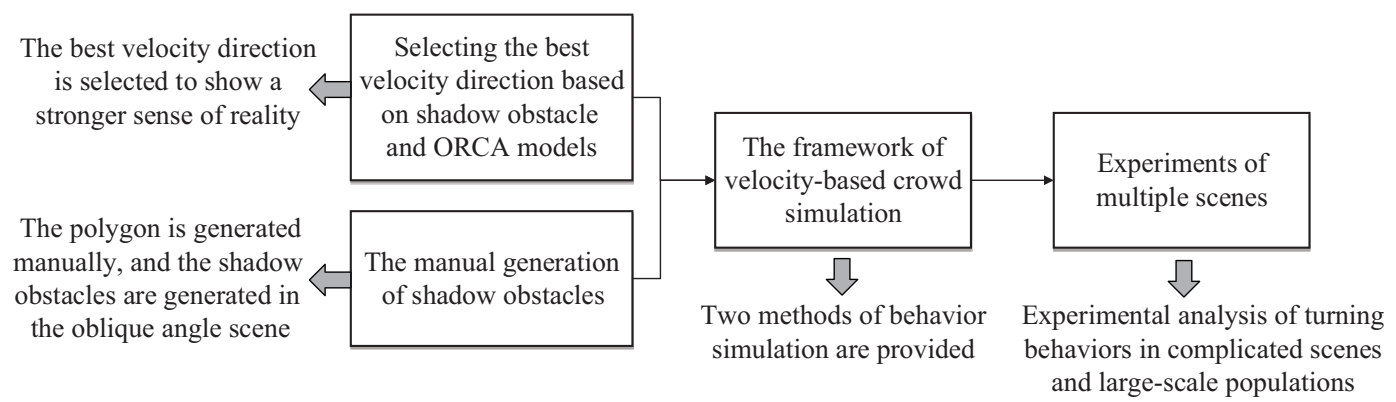

图 1 本文的总体框架图

Figure 1 The framework of this work

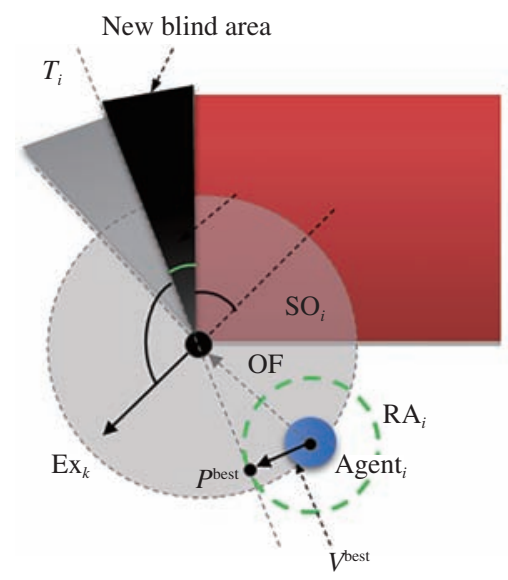

图 2 (网络版彩图) 影子障碍物中个体获得最大视野的速度方向

Figure 2 (Color online) Direction of the velocity for generating the largest viewing range using SOM

的圆形作为其形状, 并在仿真环境的每一个拐角处都放置影子障碍物用来计算和模拟行人的转弯行为, 其中拐角的定义为多边形障碍物中的凸角. 影子障碍物的影响范围 $R^{\mathrm{SO}}$ (圆的半径), 位置 $C^{\mathrm{SO}}$ (圆的 中心), 所在拐角的内角大小 $\mathrm{Ang}^{\mathrm{SO}}$; 拐角两侧平面向量 $W 1^{\mathrm{SO}}$ 和 $W 2^{\mathrm{SO}}$, 其模长分别为平面的宽度和 长度; 辅助单位向量 $\mathrm{Ex}^{\mathrm{SO}}$ 用以对拐角进行均分, 该向量可由 $W 1^{\mathrm{SO}}$ 和 $W 2^{\mathrm{SO}}$ 得出.

影子障碍物的触发条件为行人试图转过拐角时没有获得足够的拐角视野. 假设在某一个时间点 $t$, 个体 $i$ 的位置是 $p_{i}$, 速度是 $v_{i}$, 期望速度的大小是 $\mathrm{SP}_{i}^{\text {pref }}$, 同时也满足影子障碍物 $\mathrm{SO}_{k}$ 的触发条件, 影 子障碍物的位置为 $C_{k}^{\mathrm{SO}}$. 那么在接下来 $\Delta t$ 时间里, 个体 $i$ 的所有可达区域 $\mathrm{RA}_{i}$ 为一个圆形 (如图 2 所示). 根据视野最大化原则, 最佳的速度方向为 $v^{\text {best [11] }}$

$$
v^{\text {best }}=\left(C_{k}^{\mathrm{SO}}-p_{i}\right)_{\perp}^{*} \cdot \mathrm{SP}_{i}^{\text {pref }} .
$$

\subsection{ORCA 模型}

ORCA 模型 [12] 通过处理个体的避碰行为以及对应的可行速度集合, 能够有效地避免 RVO 模型 ${ }^{[23]}$ 中存在的行人抖动问题. 该模型将速度方向限定在一个平面内, 只要个体速度落到该半平面, 则可以避 免碰撞. 个体 $\mathrm{A}$ 相对于个体 $\mathrm{B}$ 的 $\mathrm{ORCA}_{\mathrm{A} / \mathrm{B}}$ 半平面定义为点 $v_{\mathrm{A}}+(1 / 2) u$, 方向为 $n$ 的半平面. 其中 


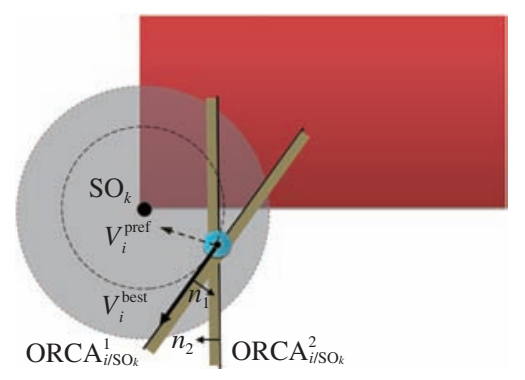

图 3 (网络版彩图) 影子障碍物模型转换为 ORCA 半平面

Figure 3 (Color online) Transformation from SOM to ORCA half plane

$u$ 的计算如下:

$$
u=\left(\arg \min _{v \in \partial \mathrm{VO}_{\mathrm{A} / \mathrm{B}}^{\tau}}\left\|v-\left(v_{\mathrm{A}}-v_{\mathrm{B}}\right)\right\|\right)-\left(v_{\mathrm{A}}-v_{\mathrm{B}}\right),
$$

其中 $n$ 为 $\mathrm{VO}_{\mathrm{A} / \mathrm{B}}^{\tau}$ 上一点 $v_{\mathrm{A}}-v_{\mathrm{B}}+u$ 的法向量, $\mathrm{VO}_{\mathrm{A} / \mathrm{B}}^{\tau}$ 表示 $\mathrm{A}$ 相对于 $\mathrm{B}$ 的 $\tau$ 时间速度障碍物.

假设 $\left\{\mathrm{CO}_{k} \mid k=1,2, \ldots, m\right\}$ 为所有静态障碍物的集合, 所有行人个体的集合为 $\left\{\mathrm{AO}_{k} \mid k=1,2, \ldots\right.$, $n\}$, 则对于个体 $i$ 而言, 总的 ORCA 集合为:

$$
\mathrm{ORCA}_{i}=\left(\bigcap_{k-1}^{m} \mathrm{ORCA}_{i \mid \mathrm{CO}_{k}}\right) \bigcap\left(\bigcap_{k-1}^{n} \mathrm{ORCA}_{i \mid \mathrm{AO}_{k}}\right) .
$$

当个体处在一个高密度的场景中时, 其某个时刻的 ORCA 半平面交集很有可能是空集, 此时通常 会选择一个惩罚最小的速度作为新的个体速度 ${ }^{[12]}$.

\section{3 基于障碍物模型的 ORCA 半平面转化方法}

影子障碍物模型获得的最佳速度方向可以使个体的转弯行为更加真实, 而 ORCA 模型是将速度 方向限定在一个半平面内, 从而实现个体的避碰. 个体的速度方向是体现仿真中个体运动是否具有真 实感的关键, 为了进一步提高仿真个体的行为真实感, 速度的选择也成为影子障碍物模型与 ORCA 模 型结合的关键. 本节通过将影子障碍物模型转化为 ORCA 半平面来实现速度的选择.

为了实现速度的选择, 本文将影子障碍物中的 $v_{i}^{\text {best }}$ (式 (1)) 转化为 ORCA 半平面, 如图 3 所示. 为将单一的速度转换为可行速度集合, 本文将该问题转换为求个体 $i$ 相对于拐角 $k$ 的 ORCA 半平面 的问题, 即如何获得个体转弯行为产生的可行速度集合. 该可行速度集合满足两个条件:

(1) 在不考虑其他可行速度集合的情况下, 从该集合中选择一个最接近 $v_{i}^{\text {pref }}$ 的速度作为 $v_{i}^{\text {best; }}$;

(2) 该速度集合是个体避碰行为可行速度集合的超集，即该速度集合不会影响避碰行为的速度 选择.

由于 $v^{\text {best }}=\left(C_{k}^{\mathrm{SO}}-p_{i}\right)_{\perp}^{*} \cdot \mathrm{SP}_{i}^{\mathrm{pref}}$, 其中 $\left(C_{k}^{\mathrm{SO}}-p_{i}\right)_{\perp}^{*}$ 是一个单位向量, 条件 (1) 可以适当减弱条件 为选择速度的方向与 $v_{i}^{\text {best }}$ 一致. 对于条件 (2), 由于个体避碰行为中可行速度集合的不确定性, 本文 将首先计算除去影子障碍物 ORCA 的集合, 即根据式 (3) 的可行速度集合, 计算出个体新的速度, 然 后将新的速度 $v_{i}^{\text {new }}$ 与目前的期望速度 $v_{i}^{\text {pref }}$ 进行比较. 假如速度方向的夹角大于一定值, 则表示其他 障碍物对个体有一定的影响, 此时将直接使用 $v_{i}^{\text {new }}$ 作为新的速度方向. 否则, 根据加入影子障碍物的 ORCA 集合更新 $v_{i}^{\text {new }}$ 并作为新的速度方向. 


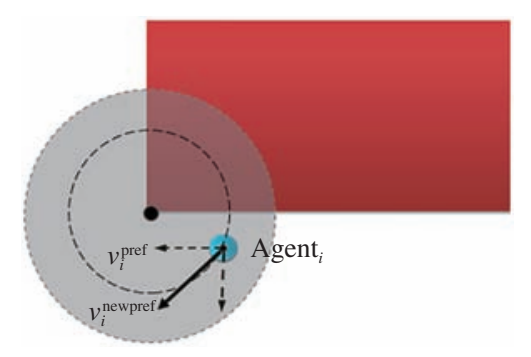

图 4 (网络版彩图) 根据离墙心理的期望速度计算

Figure 4 (Color online) Preferred velocity calculation based on the psychology of keeping a distance with walls

为了满足条件 (1), 本文利用两个 ORCA 半平面来表示个体转弯行为的可行速度集合, 如图 3 所 示. 其中一个 $\mathrm{ORCA}$ 半平面过原点, 方向 $n_{1}$ 垂直于 $v_{i}^{\text {best }}$. 另一个 $\mathrm{ORCA}$ 半平面同样经过原点, 方向 $n_{2}$ 平行于离个体较近的墙面方向. 如果个体 $i$ 受到影子障碍物的影响, 则他的期望速度不在上述两个 ORCA 平面形成的可行速度集合中, 因此, 在选择最接近期望速度的速度时, 必定会选择该集合边界 上的速度. 又因为个体期望速度与 $\mathrm{ORCA}_{i / \mathrm{SO}_{k}}^{1}$ 的夹角小于个体期望速度与 $\mathrm{ORCA}_{i / \mathrm{SO}_{k}}^{2}$ 的夹角, 因此 选择的速度方向即为 $v_{i}^{\text {best }}$ 的速度方向.

\section{4 基于障碍物模型的期望速度计算方法}

转弯行为的心理约束影响着个体期望速度的选择 ${ }^{[11]}$, 即个体会在以最短路径达到目标和以某种 安全的策略到达目标中做出权衡, 本文选用了线性插值的方法进行权衡.

如图 4 所示, 假设个体 $i$ 的原期望速度为 $v_{i}^{\text {pref }}$, 场景中所有影子障碍物的集合为 $\left\{\mathrm{SO}_{k} \mid k=\right.$ $1,2, \ldots, m\}$, 影子障碍物 $\mathrm{SO}_{k}$ 引导的速度方向为 $v_{i}^{\mathrm{SO}_{k}}$, 那么该个体新的期望速度为

$$
v_{i}^{\text {newpref }}=\delta \cdot \sum_{k=1}^{m} v_{i}^{\mathrm{SO}_{k}}+(1-\delta) \cdot v_{i}^{\text {pref }}
$$

其中 $v_{i}^{\mathrm{SO}_{k}}$ 即式 (2) 中的 $v_{i}^{\text {best }}, \delta \in[0,1]$ 为影子障碍物模型中定义的个性因子. $\delta$ 越大说明个体的安全 意识越高, 受到影子障碍物模型的影响越大, 即转弯行为相对目标行为的比重越大. 这种基于 ORCA 速度半平面选择个体新期望速度的方法能够较好地将影子障碍物与原始 ORCA 模型进行结合, 并且 计算开销相对较小.

\section{4 影子障碍物的手动生成方法}

影子障碍物模型采用包围盒生成有效拐角, 这种自动生成的方法只能处理拐角为直角的情况. 为 了解决该问题, 本文在自动生成影子障碍物的基础上用多边形代替包围盒, 在封闭墙面和非封闭墙面 的凸角处均能够生成影子障碍物. 如图 5 所示, 首先根据自定义需求手动创建多边形, 然后根据多边 形的顶点信息生成对应的影子障碍物. 由于影子障碍物的拐角是多边形的凸角, 因此在生成影子障碍 物的过程中需要将凹点去除, 如图 5(b) 所示.

对于非封闭型的墙面, 生成影子障碍物的方法基本和封闭型的情况相同, 如图 6 所示. 此时需要 注意的是, 我们认为拐角内角在折线段的两个端点处的大小为 0 , 因此对应影子障碍物的辅助向量如 图 6(b) 所示. 另外, 由于在这种情况下, 个体可以位于墙面的两侧 (图 6 (c) 所示), 因此需要判定个体 


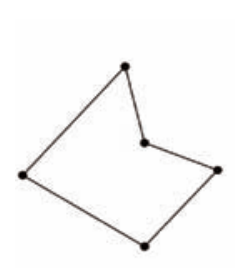

(a)

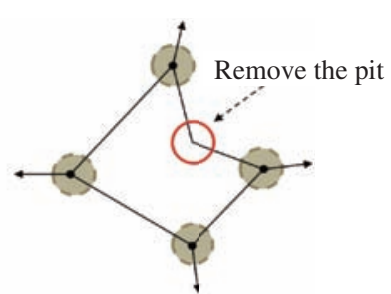

(b)

图 5 (网络版彩图) 封闭型墙面情况下生成影子障碍物方法

Figure 5 (Color online) The generation of the SOM with enclosed walls. (a) Manual-generated polygon; (b) generating shadow obstacle corresponding to vertex information

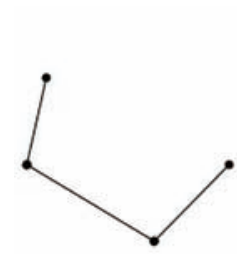

(a)

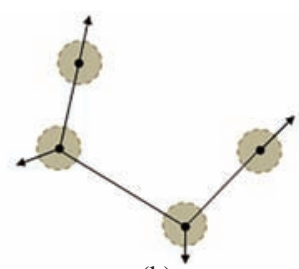

(b)

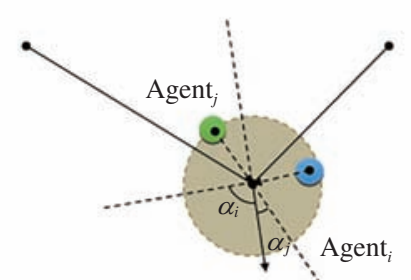

(c)

图 6 (网络版彩图) 非封闭型墙面情况生成影子障碍物方法

Figure 6 (Color online) The generation of the shadow obstacles with non-closed walls. (a) Representation of non-closed walls; (b) generating shadow obstacles; (c) the method to determine the position of the agents

在凸角一侧还是在凹角一侧. 本文通过个体位置 $p_{i}$ 到影子障碍物中心点 $C^{\mathrm{SO}}$ 的向量与影子障碍物辅 助向量 $\mathrm{Ex}^{\mathrm{SO}}$ 间的夹角 $\alpha$ 进行判定: 若 $\alpha$ 小于拐角内角的一半, 则认为个体在凹角一侧, 此时该个体 不受影子障碍物影响.

\section{5 基于速度的人群仿真框架}

传统的人群仿真系统 ${ }^{[7,33,34]}$ 通常较少考虑物理力的作用, 并且一般的物理模拟系统无法模拟个 体产生的抑制力. 因此, 本文将使用游戏引擎中的角色控制器 [35] 组件模拟个体, 并且利用物理系统来 模拟一般障碍物. 运动模拟真实感及计算效率均为评价仿真系统优劣的重要指标. 为了提高运行效率 和更新效率, 本文选用了角色控制器对个体进行物理模拟, 并在具体更新个体信息时, 根据心理作用 力 $F^{\text {psy }}$ 计算出个体下一帧的速度.

本文提出的基于速度的人群仿真框架如图 7 所示, 它依据个体的心理变化构筑一系列的速度约束 条件, 个体将根据这些约束条件选择新的速度行进. 框架共提供两种行为模拟方法, 一种是全局路径 规划与 ORCA 模型结合的方法, 另一种是影子障碍物模型与 ORCA 模型结合的方法. 这两种模拟方 法分别利用全局路径规则算法和影子障碍物模型对个体目标行为进行处理, 求得个体的期望速度 $v_{i}^{\text {pref }}$ 之后, 再利用 ORCA 模型对个体避碰行为进行处理, 最后求得所有可行的速度集合. 如果可行速度集 


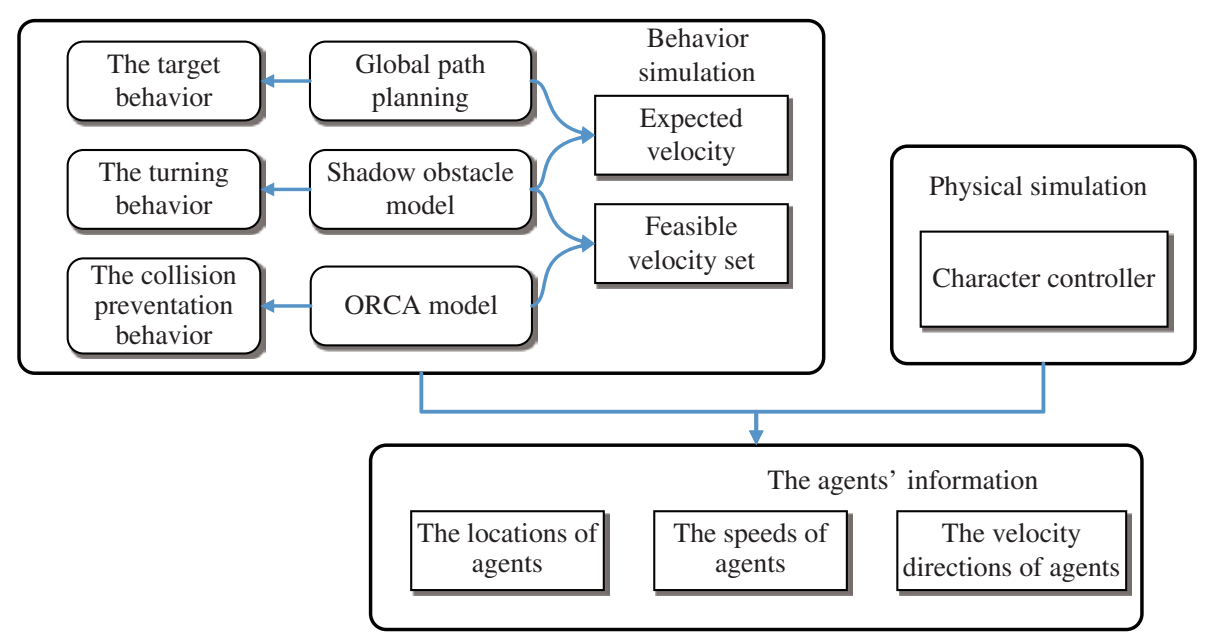

图 7 (网络版彩图) 基于速度的人群仿真框架

Figure 7 (Color online) The framework of velocity-based crowd simulation

合非空, 则在该集合中选择一个最接近 $v_{i}^{\text {pref }}$ 的速度作为个体新的速度方向; 如果可行速度集合为空, 则在可行速度集合中选择一个惩罚最小的速度 ${ }^{[12]}$ 作为个体新的速度, 之后在更新个体信息时, 将新 的速度输入到个体的角色控制器中.

\section{6 实验结果与分析}

本文将对相对复杂的场景以及大规模人群的转弯行为进行实验分析: 通过 Unity3D 引擎构建了 拐角、通道、多障碍物, 以及地铁站复杂场景, 用以评估本文提出的行人仿真算法; 与基于社会力的模 型进行对比, 展示本文所提方法的有效性.

\section{1 拐角轨迹分析}

本小节将对本文所提方法与 ORCA 模型在拐角处的转弯场景进行比较, 行人在拐角处的运动轨 迹如图 8 所示. 由式 (4) 可知, 当 $\delta=0$ 时, 模型的速度结果是原始的 ORCA 模型计算出的速度, 如 图 8(a) 所示. 在该情况下, ORCA 模型作用的个体始终选择最短路径行进, 因此个体在拐角处的轨迹 显得比较生硬, 不符合真实人在转弯时具有的离墙心理. 从图 8(b) 和 (c) 中可以看出, 当 $\delta$ 增大时, 个 体离墙的距离变大, 并且在拐角处的轨迹曲线更加圆滑. 由于目前的参数均为手动选择, 导致轨迹有 时显得不够自然, 因此在后续的研究中还将采用数据驱动 ${ }^{[36,37]}$ 的方法来拟合参数并进行改良.

\section{2 狭窄通道分析}

本小节将分析比较在狭窄通道场景中本文方法与基于社会力模型的行人运动情况, 比较结果如 图 9 所示. 基于社会力的人群仿真模型和本文所提的人群仿真模型都可以很好地模拟狭窄通道场景, 二者在通道口均未出现阻塞现象. 但是相对于社会力模型来说, 本文所提的方法能够更好地模拟出行 人的自组织现象, 即行人能够自发地使局部区域达到有序. 其中个性因子取值越大, 自组织现象相对更 加明显, 如图 9 (b) 中红框所示. 而在基于社会力的仿真模拟结果中, 行人分布比较零散, 如图 9(a) 所 示; 同时在模拟过程中, 行人时常会出现抖动现象从而导致模拟效果失真. 


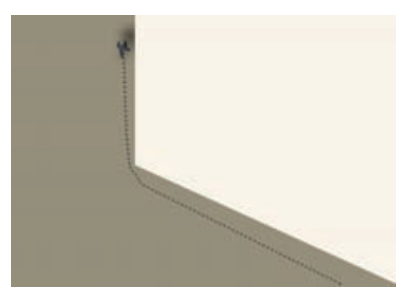

(a)

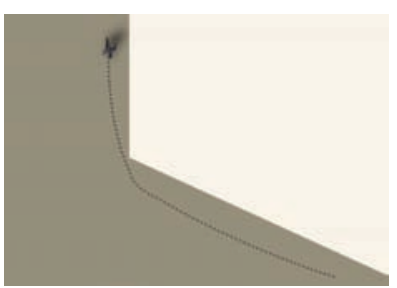

(b)

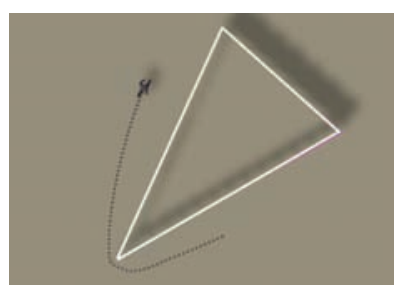

(c)

图 8 (网络版彩图) 任意角度拐角行人运动轨迹

Figure 8 (Color online) Trajectories of the pedestrian in arbitrary convex corner. (a) Obtuse angle with $\delta=0$; (b) obtuse angle with $\delta=0.25 ;$ (c) acute angle with $\delta=0.25$

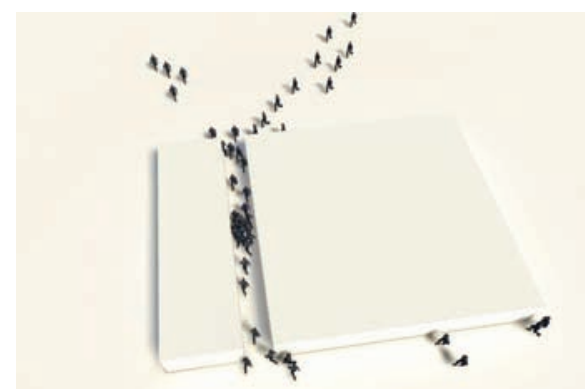

(a)

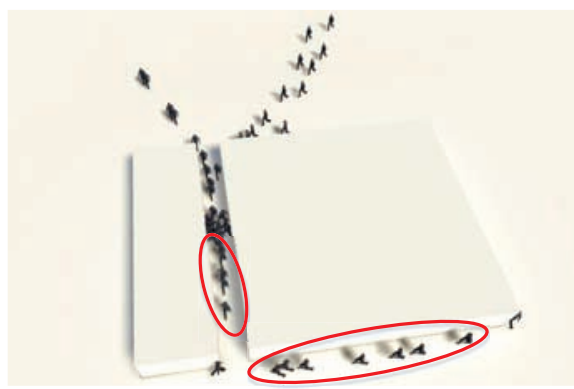

(b)

图 9 (网络版彩图) 狭窄通道场景模拟结果图

Figure 9 (Color online) Simulation result of narrow passage scenario. (a) Force-based crowd simulation model; (b) velocity-based crowd simulation model with $\delta=0.2$

\section{3 多障碍物复杂场景}

为了分析本文算法在复杂场景下的仿真效果, 本小节比对了在多障碍物的复杂场景中不同人数、 不同个性因子对个体运动状态的影响. 图 10(a) 和 (d) 是人数分别为 16 和 48 人的初始场景, 在开始 模拟之后, 每个个体的目标点均设定为其对角线的位置, 即在对角线位置的个体以场景中心为原点相 互交换位置. 图 10(b) 和 (e) 所示为 16 和 48 人时 $\delta=0$ 的场景, 也是 ORCA 原始模型, 模拟结果显 示, 该情况模拟出的行人运动轨迹不够平滑, 拐角处行人运动轨迹也不够自然. 而当 $\delta=0.25$ 时, 如 图 10(c) 和 (f) 所示, 本文提出的影子障碍物与 ORCA 模型结合的方法模拟出的行人轨迹更加平滑, 并且行人有向走廊中间聚集并逐渐达到有序的现象, 如图中虚线椭圆的标识, 该现象更加符合实际 情况.

\section{4 复杂地铁站场景}

本文构建了复杂的地铁站场景, 并对本文所提方法以及基于力的模型在仿真效果及计算效率两方 面进行了比较. 由于地铁站场景中存在大量的多层结构, 仿真个体上下楼梯时的行为处理成为研究的 关键. 为了解决该问题, 本文将个体的位置统一映射到同一高度的平面, 然后再在该平面上应用人群仿 真算法. 地铁站场景的模拟结果如图 11 所示, 两种模型在楼梯口均有部分拥堵现象, 拥堵的原因在于 上下楼梯的处理. 由于本文采用了位置映射, 因此行人在避碰时会引入一些原本不需要考虑避碰的个 体, 虽然出现部分拥堵, 但是所有个体都顺利地通过了楼梯口, 没有出现完全堵死的现象.

为测试本文模型的计算效率, 将分别测试该场景中不同行人数量对每秒处理帧数的影响. 测试机 


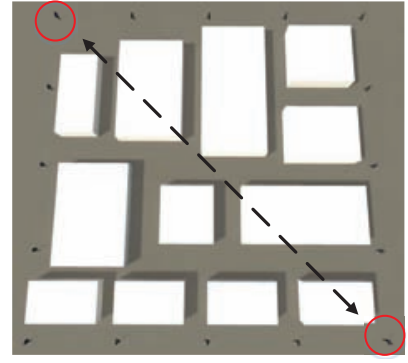

(a)

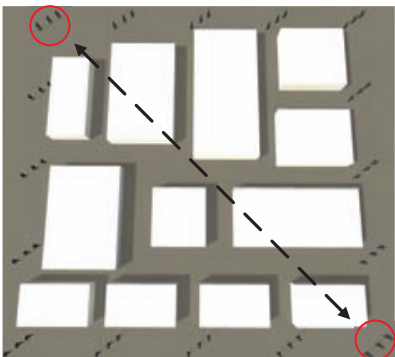

(d)

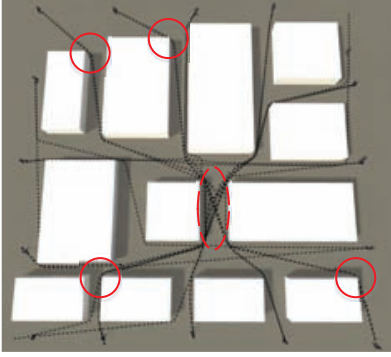

(b)

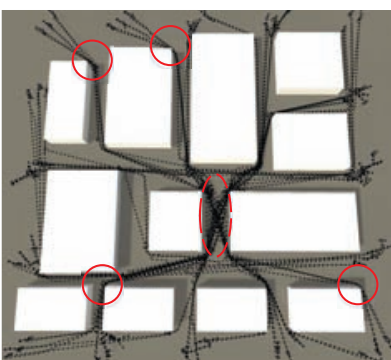

(e)

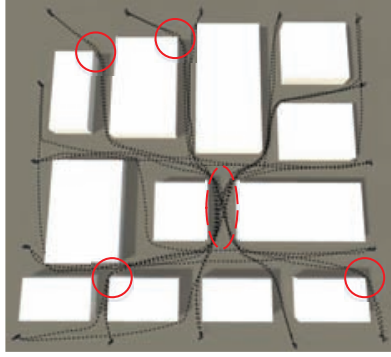

(c)

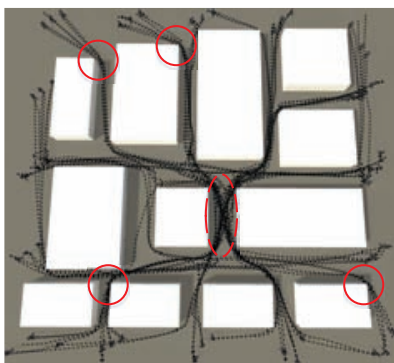

(f)

图 10 (网络版彩图) 多障碍物复杂场景模拟结果图

Figure 10 (Color online) Simulation result of complex scene with many obstacles. (a) Initial state with 16 agents; (b) result with $\delta=0$ and 16 agents; (c) result with $\delta=0.25$ and 16 agents; (d) initial state with 48 agents; (e) result with $\delta=0$ and 48 agents; (f) result with $\delta=0.25$ and 48 agents

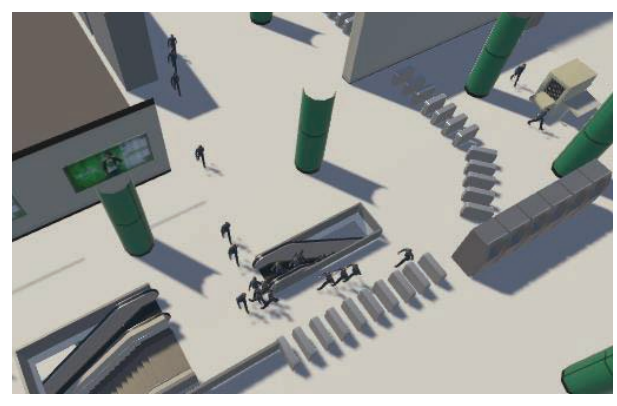

(a)

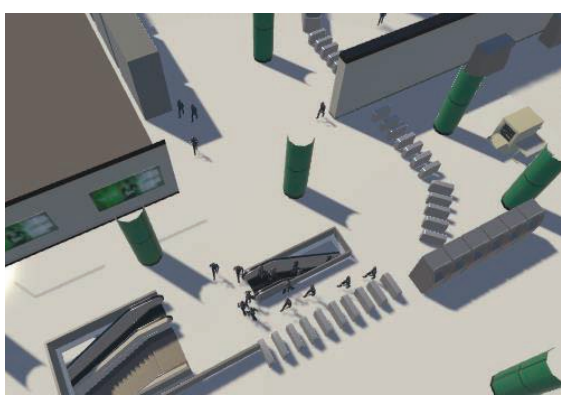

(b)

图 11 (网络版彩图) 复杂地铁站场景模拟结果图

Figure 11 (Color online) Simulation result of complex subway station scenario. (a) Our approach; (b) force-based crowd simulation model

表 1 复杂地铁站场景性能测试结果

Table 1 Performance test result in complex subway station scenario

\begin{tabular}{ccc}
\hline The number of agents & Social force model (fps) & The proposed model (fps) \\
\hline 40 & $\approx 67$ & $\approx 70$ \\
100 & $\approx 10$ & $\approx 45$ \\
150 & $\approx 3$ & $\approx 25$ \\
\hline
\end{tabular}

器配置为 CPU: $2.5 \mathrm{GHz}$ 双核, GPU: GT755M. 程序在单线程模式下运行, 测试结果如表 1 所示.

测试结果显示, 基于社会力的模型在场景人数较少时和本文提出模型的帧率相当. 当场景人数达 

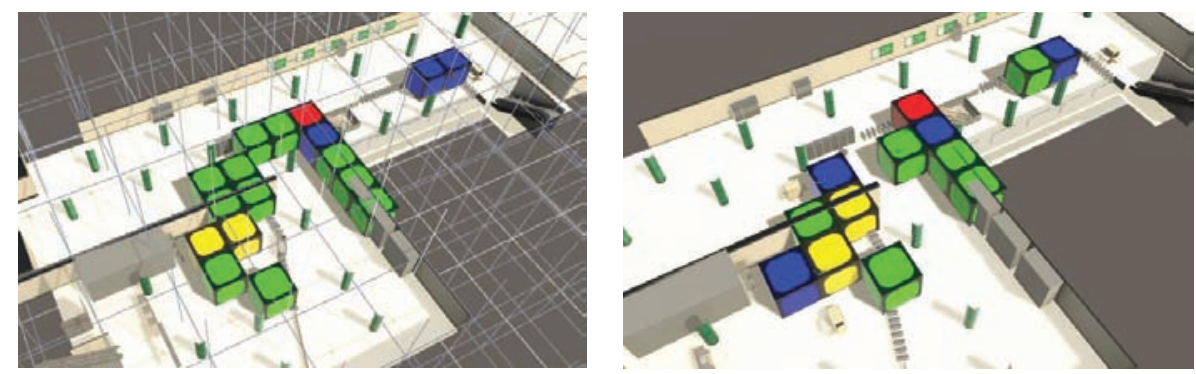

图 12 (网络版彩图) 体素化场景与空间数据分析结果

Figure 12 (Color online) Voxelization of the scene and the result of data analysis

到一定数量后, 基于社会力模型的帧率急速下降, 无法支持程序的流畅运行. 这是因为社会力模型需 要计算场景中仿真个体之间的相互作用力, 其计算量与仿真人数 $n$ 的平方成正比; 而本文所提方法仅 对仿真个体本身进行速度方向的计算, 其计算量与场景中的仿真人数 $n$ 成正比, 因此本文所提的模型 在人群数量较多的大规模场景中同样具有较好的计算性能.

\section{5 疏散演练评估系统}

由于实景演练中的数据采集困难、场景真实感对人主观判断的影响较大, 使得演练结果存在一定 的误差. 本小节将影子障碍物与 ORCA 模型结合的方法应用在疏散演练评估系统的行人仿真运动中, 在演练过程中, 指导者可以及时分析数据, 并在演练结束后, 通过添加空间数据分析功能帮助指导者 评估疏散的结果. 最后, 本文还使用 Unity3D 系统制作高度真实感的场景, 通过对场景真实感渲染, 以 最大程度模拟真实环境对行人主观判断的影响.

(1) 空间数据分析. 本系统的疏散演练场景是三维多层次场景, 为了使指导者对演练场景某区域的 疏散人员混乱程度和撤离情况具有更直观的感受, 本文采用将空间体素化的方法划分区域, 如图 12 所 示. 在模拟的过程中实时记录空间小立方体中的个体信息, 并利用不同颜色的方块表示区域个体数量 以及区域内部个体速度一致性. 本文采用 Vicsek 等 ${ }^{[38]}$ 提出的方法对个体速度的一致性 $\bar{v}$ 进行计算:

$$
\bar{v}=\frac{1}{N}\left|\sum_{i=1}^{N} \frac{v_{i}}{\left|v_{i}\right|}\right|,
$$

其中 $N$ 表示区域内个体的数量, $v_{i}$ 表示区域内个体 $i$ 的速度与方向, $\left|v_{i}\right|$ 表示个体速度的绝对值. 由 式 (5) 所示, 当区域内个体速度的一致性越高, $\bar{v}$ 的值越趋近于 1 , 反之 $\bar{v}$ 的值越趋近于 0 . 在本文的 场景分析中, 区域内的空间小立方体的颜色根据个体速度一致性 $\bar{v} \in[0,1]$ 的值由小到大分别设置为 红色、黄色、蓝色和绿色.

(2) 真实感场景渲染. 对于疏散演练系统而言, 模拟环境是否身临其境对人的主观判断会起到很大 影响. 本文参考 Unity3D 官方提供的简单材质属性对应表, 对 Shader 的 Albedo, Metallic, Smoothness 3 个属性进行设定, 更加接近现实生活中的材质属性, 从而达到较好的材质效果. 除此之外, 本文还选 用 Unity3D 中烘焙全局光照贴图的处理方法来模拟光的互动和反弹等行为.

图 13 所示为使用全局光照前后的效果对比. 在使用了全局光照后, 场景的光影质量有了显著提 高. 但是在计算全局光照的时候, 缺少场景中材质的镜面反射信息, 因此本文使用 Unity3D 提供的 reflection probe, 即使用环境贴图 (cubemap) ${ }^{[39]}$ 的形式记录下物体周围的环境信息. 图 14 所示的渲染 结果中, 线框中的柱子使用了 reflection probe 的信息之后具有比较明显的镜面反射效果. 


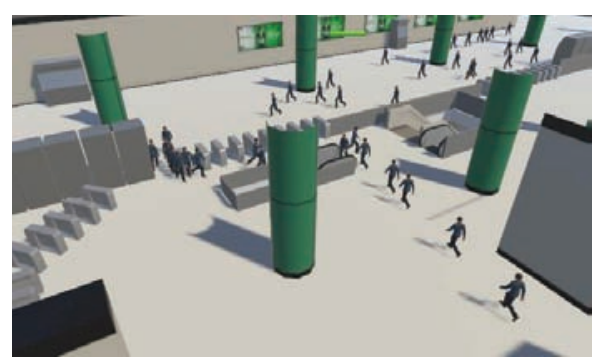

(a)

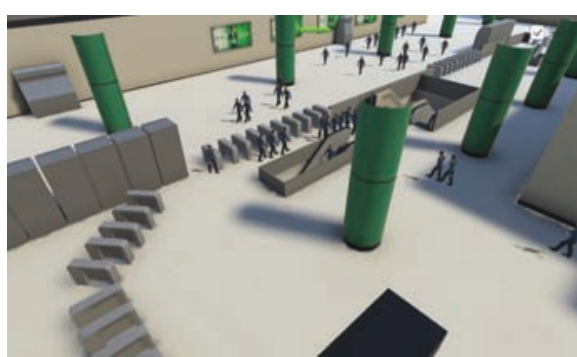

(b)

图 13 (网络版彩图) 全局光照场景渲染前后对比

Figure 13 (Color online) Comparison of the scene before and after global illumination rendering. (a) Before rendering; (b) after rendering
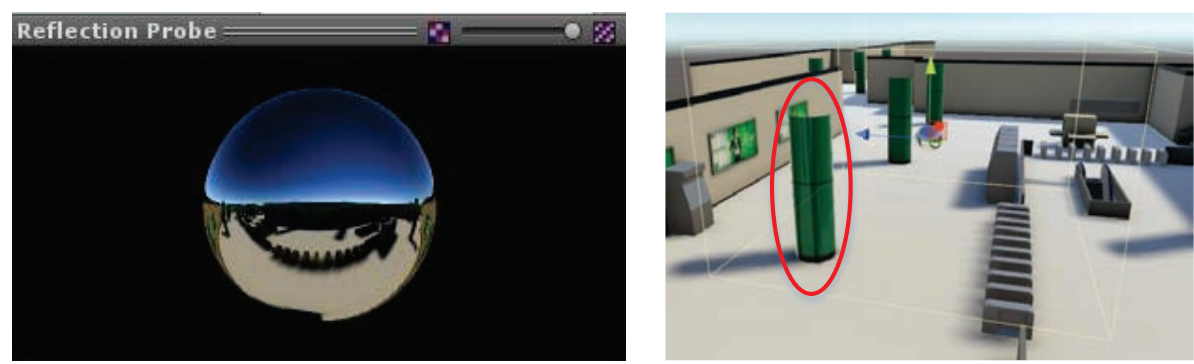

图 14 (网络版彩图) 利用 reflection probe 生成镜面反射信息

Figure 14 (Color online) Generation of reflection information using reflection probe

\section{7 总结与展望}

本文从用户的安全心理特征和运动速度特征出发, 提出了结合影子障碍物和 ORCA 模型的人群 仿真方法. 首先本文将影子障碍物模型转化为 ORCA 半平面和期望速度, 分别提出了两种影子障碍物 和 ORCA 模型的结合方法. 然后提出了影子障碍物的手动生成方法, 用以处理任意角度的拐角以及一 些非封闭墙面的情况. 最后, 本文融合行为模拟和物理模拟技术, 构建了基于速度的人群仿真框架, 对 复杂场景中大规模人群进行行为仿真分析, 并采用 Unity3D 引擎对地铁站场景进行了真实感渲染. 实 验结果表明本文所提方法均能较好地模拟各类拐角处的人群行为, 并且方法具有较高的计算效率. 在 未来的研究中, 将深入研究人群仿真轨迹的模拟, 通过将集聚人群的有序性特征加入到虚拟现实的沉 浸式演练系统中, 进一步增强人群模拟的真实感.

\section{参考文献}

1 Nasir F M, Sunar M S. A survey on simulating real-time crowd simulation. In: Proceedings of International Conference on Interactive Digital Media, Bandung, 2016. 1-5

2 Xu M L, Jiang H, Jin X G, et al. Crowd simulation and its applications: recent advances. J Comput Sci Technol, 2014, 29: 799-811

3 Alanjari P, Razavialavi S R, Abourizk S. Material and facility layout planning in construction projects using simulation. In: Proceedings of the 2014 Winter Simulation Conference, Savannah, 2014. 3388-3398

4 Mitsuhara H, Inoue T, Yamaguchi K, et al. Game-based evacuation drill inside google street view. In: Advances in Human Factors, Business Management, Training and Education. Berlin: Springer, 2017. 655-666

5 Veziridis S, Karampelas P, Lekea I. Learn by playing: a serious war game simulation for teaching military ethics. In: 
Proceedings of IEEE Global Engineering Education Conference, Athens, 2017. 920-925

6 Zhang D, Xie Z, Li P, et al. Real-time navigation in dynamic human environments using optimal reciprocal collision avoidance. In: IEEE International Conference on Mechatronics and Automation, Beijing, 2015. 2232-2237

7 Helbing D, Molnár P. Social force model for pedestrian dynamics. Phys Rev E, 1995, 51: 4282

8 Helbing D, Farkas I, Vicsek T. Simulating dynamical features of escape panic. Nature, 2000, 407: 487-490

9 Cui X, Shi H. An overview of pathfinding in navigation mesh. Int J Comput Sci Netw Secur, 2012, 12: 48-51

10 Hashimoto K, Yoshimi T, Mizukawa M, et al. A study of collision avoidance between service robot and human at corner-analysis of human behavior at corner. In: Proceedings of International Conference on Ubiquitous Robots and Ambient Intelligence, Jeju, 2013. 383-384

11 He G Q, Jin Y, Chen Q, et al. Shadow obstacle model for realistic corner-turning behavior in crowd simulation. Front Inf Technol Elect Eng, 2016, 17: 200-211

12 Berg J V D, Guy S, Lin M, et al. Reciprocal n-body collision avoidance. In: Robotics Research. Berlin: Springer, 2011. $3-19$

13 Zhou S P, Chen D, Cai W T, et al. Crowd modeling and simulation technologies. ACM Trans Model Comput Simulation, 2010, 20: 1-35

14 Lakoba T I, Kaup D J, Finkelstein N M. Modifications of the helbing-molnár-farkas-vicsek social force model for pedestrian evolution. Simulation, 2005, 81: 339-352

15 Qu Y C. Modeling evacuation behavior and analyses of dynamic behavior characteristics of crowd. Dissertation for Ph.D. Degree. Beijing: Beijing Jiaotong University, 2015 [屈云超. 密集人群疏散行为建模与动态特性研究. 博士学 位论文. 北京: 北京交通大学, 2015]

16 Karamouzas I, Overmars M. Simulating and evaluating the local behavior of small pedestrian groups. IEEE Trans Visual Comput Graph, 2012, 18: 394-406

17 Reynolds C W. Flocks, herds, and schools: a distributed behavioral model. Comput Graph, 1987, 21: 25-34

18 Reynolds C W. Steering behaviors for autonomous characters. In: Proceedings of Game Developers Conference, San Francisco, 1999

19 Hu Q M, Fang W N, Jia Y Q, et al. Study of crowded environment and cluster behavior simulation. Sci China Ser E-Technol Sci, 2009, 39: 1034-1038 [胡清梅, 方卫宁, 贾玉泉, 等. 人群拥挤及群集行为特性的仿真研究. 中国科学 E 辑: 技术科学, 2009, 39: 1034-1038]

20 Pelechano N, Allbeck J M, Badler N I. Controlling individual agents in high-density crowd simulation. In: Proceedings of the 2007 ACM SIGGRAPH/Eurographics Symposium on Computer Animation, San Diego, 2007. 99-108

21 Moussaid M, Helbing D, Theraulaz G. How simple rules determine pedestrian behavior and crowd disasters. Proc Natl Acad Sci, 2011, 108: 6884-6888

22 Kremyzas A, Jaklin N, Geraerts R. Towards social behavior in virtual-agent navigation. Sci China Inf Sci, 2016, 59: 112102

23 Berg J V D, Lin M, Manocha D. Reciprocal velocity obstacles for real-time multi-agent navigation. In: Proceedings of IEEE International Conference on Robotics and Automation, Pasadena, 2008. 1928-1935

24 Golas A, Narain R, Curtis S, et al. Hybrid long-range collision avoidance for crowd simulation. IEEE Trans Visual Comput Graph, 2014, 20: 1022-1034

$25 \mathrm{Wu}$ Q Q, Ji Q G, Du J H, et al. Simulating the local behavior of small pedestrian groups using synthetic-vision based steering approach. In: Proceedings of the 12th ACM SIGGRAPH International Conference on Virtual-Reality Continuum and Its Applications in Industry, Hong Kong, 2013. 41-50

26 Jan O. A synthetic-vision based steering approach for crowd simulation. ACM Trans Graph, 2010, 29: 1-9

27 Suzuki T, Takahashi M. Obstacle avoidance for autonomous mobile robots based on position prediction using fuzzy inference. In: Proceedings of the International Conference on Informatics in Control, Automation and Robotics, Volume Robotics and Automation, Milan, 2009. 299-304

28 Yeh H, Curtis S, Patil S, et al. Composite agents. In: Proceedings of the ACM SIGGRAPH/Eurographics Symposium on Computer Animation, Dublin, 2008. 39-47 
29 Wang H, Xu M L, Zhu F B, et al. Shadow traffic: a unified model for abnormal traffic behavior simulation. Comput Graph, 2018, 70: 235-241

30 Watt A. 3D Computer Graphics. Boston: Addison-Wesley Longman Publishing, 1993

31 Snook G. Simplified 3d movement and pathfinding using navigation meshes. In: Game Programming Gems. Hingham: Charles River Media, 2000. 288-304

32 Berg J V D, Guy S J, Lin M, et al. Reciprocal n-body collision avoidance. In: Robotics Research. Berlin: Springer, 2011. $3-19$

33 Hughes R L. The flow of hunman crowds. Annu Rev Fluid Mech, 2003, 35: 169-182

34 Reynolds C W. Steering behaviors for autonomous characters. In: Proceedings of Game Developers Conference, San Francisco, 1999. 763-782

35 Unity Technologies. Unity user manual. 2017.2-001R. 2017. http://docs.unity3d.com/Manual/class-Character Contro ller.html

36 Guy S J, Kim S, Lin M C, et al. Simulating heterogeneous crowd behaviors using personality trait theory. In: Proceedings of the ACM SIGGRAPH/Eurographics Symposium on Computer Animation, Vancouver, 2011. 43-52

37 Kang H L, Choi M G, Hong Q, et al. Group behavior from video:a data-driven approach to crowd simulation. In: Proceedings of the ACM SIGGRAPH/Eurographics Symposium on Computer Animation, San Diego, 2007. 109-118

38 Vicsek T, Czirok A, Benjacob E, et al. Novel type of phase transition in a system of self-driven particles. Phys Rev Lett, 1995, 75: 1226-1229

39 Greene N. Environment mapping and other applications of world projections. IEEE Comput Graph Appl, 1986, 6: $21-29$

\title{
Crowd behavior simulation based on shadow obstacle and ORCA models
}

\author{
Gaoqi $\mathrm{HE}^{1,2}$, Dongxu JIANG ${ }^{1}$, Yi JIN ${ }^{1}$, Qi CHEN ${ }^{1}$, Xingjian LU ${ }^{1,3^{*}} \&$ Mingliang XU ${ }^{4}$ \\ 1. Department of Computer Science and Engineering, East China University of Science and Technology, Shanghai \\ 200237, China; \\ 2. Key Laboratory of Geographic Information Science, Ministry of Education, East China Normal University, \\ Shanghai 200241, China; \\ 3. Smart City Collaborative Innovation Center, Shanghai Jiao Tong University, Shanghai 200240, China; \\ 4. School of Information Engineering, Zhengzhou University, Zhengzhou 450000, China \\ * Corresponding author. E-mail: luxj@ecust.edu.cn
}

\begin{abstract}
Crowd behavior simulation has become one of the key support technologies for evacuation drills and safety monitoring. However, complex psychology, personality traits and interactions among individuals increase the challenges of creating realistic crowd behavioral simulations. In this paper, we propose a crowd behavior simulation method to simulate crowd behaviors by combining the shadow obstacle model (SOM) and optimal reciprocal collision avoidance (ORCA) model. First, the SOM is converted to the expected speed, as well as the half plane of ORCA. Then, a manual generation method of SOM is proposed to simulate the crowd behaviors in corners or beside a non-closed wall. Finally, we combine behavior simulation and physical simulation techniques, and propose a velocity-based crowd simulation framework. To test the effectiveness of our proposed method, several simulation methods are implemented in a Unity3D engine-based crowd simulation in subway station environment, and the comparison results demonstrate that our proposed method outperforms force-based simulation methods.
\end{abstract}

Keywords behavior simulation, shadow obstacle model, ORCA model, Unity3D, scene realistic rendering 


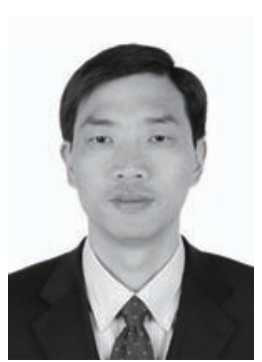

Gaoqi HE received his B.S. and M.S degrees from the Department of Mathematics in East China Normal University in 1997 and 2000, respectively. $\mathrm{He}$ received his Ph.D. degree from State Key Laboratory of CAD\&CG in Zhejiang University in 2007. He is currently an associate professor at the Department of Computer Science and Engineering at East China University of Science and Technology. From 2012 to 2013, he was a visiting associate professor at Cornell University. His research interests include video processing, crowd simulation, virtual reality and augmented reality.

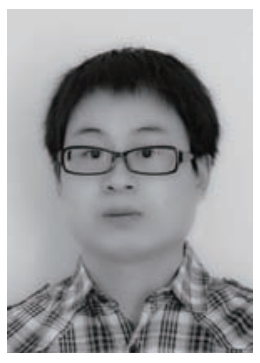

Xingjian LU is an assistant professor in the School of Information Science and Engineering, East China University of Science and Technology, Shanghai, China. He received his Ph.D. degree in Zhejiang University, China in 2014. His current research interests include cloud computing, data center, energy management, performance evaluation, and optimal scheduling for different types of workloads. E-mail: luxj@ecust.edu.cn.

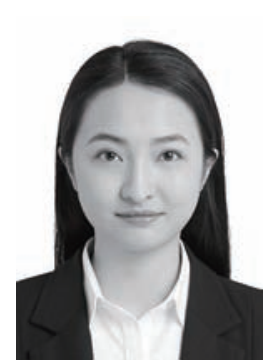

Dongxu JIANG is a postgraduate student in the School of Information Science and Engineering, East China University of Science and Technology, Shanghai, China. Her current research interests include velocity detection, abnormal detection and crowd analysis. Email: dx_harjol@163.com.

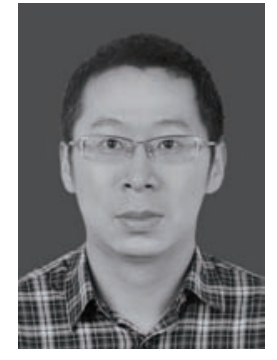

Mingliang XU received his Ph.D. degree in computer science and technology from the State Key Lab of CAD\&CG at Zhejiang University. He is a professor in the School of Information Engineering of Zhengzhou University, China, and currently is the director of CIISR (Center for Interdisciplinary Information Science Research) and the general secretary of ACM SIGAI China. His research interests include computer graphics and artificial intelligence. 